\title{
Opportunistic Strains of Saccharomyces cerevisiae: A Potential Risk Sold in Food Products
}

\author{
Roberto Pérez-Torrado ${ }^{\dagger}$ and Amparo Querol *t \\ Food Biotechnology Department, Instituto de Agroquímica y Tecnología de los Alimentos - Consejo Superior de \\ Investigaciones Científicas, Valencia, Spain
}

In recent decades, fungal infections have emerged as an important health problem associated with more people who present deficiencies in the immune system, such as HIV or transplanted patients. Saccharomyces cerevisiae is one of the emerging fungal pathogens with a unique characteristic: its presence in many food products. S. cerevisiae has an impeccably good food safety record compared to other microorganisms like virus, bacteria and some filamentous fungi. However, humans unknowingly and inadvertently ingest large viable populations of S. cerevisiae (homebrewed beer or dietary supplements that contain yeast). In the last few years, researchers have studied the nature of $S$. cerevisiae strains and the molecular mechanisms related to infections. Here we review the last advance made in this emerging pathogen and we discuss the implication of using this species in food products.

Reviewed by

Catarina Prista,

Instituto Superior de Agronomia, Portugal

Matthias Sipiczki,

University of Debrecen, Hungary

*Correspondence:

Amparo Querol

aquero/@iata.csic.es

tThese authors have contributed equally to this work

Specialty section:

This article was submitted to Food Microbiology,

a section of the journal

Frontiers in Microbiology

Received: 15 October 2015 Accepted: 17 December 2015

Published: 08 January 2016

Citation:

Pérez-Torrado R and Querol A (2016) Opportunistic Strains of Saccharomyces cerevisiae:

A Potential Risk Sold in Food

Products. Front. Microbiol. 6:1522. doi: 10.3389/fmicb.2015.01522

\section{Keywords: yeast, S. cerevisiae, food, opportunistic, infection}

\section{INTRODUCTION}

Fungal infections are an extremely important health problem. According to numerous studies, Candida albicans and other Candida species are the most remarkable pathogenic fungi which cause some 7000-28000 nosocomial infections annually (Pfaller and Diekema, 2007). The general characteristic of fungal infection is that it is produced as a result of reduced immunity. Most fungal pathogens are classified as opportunistic. This concept implies that under normal conditions, these organisms are not capable of producing infection but, when host defenses are weakened, there is room for them to prosper and to generate a health problem. Another general characteristic of fungal infections is that they are frequently moderated and localized. However, fungal pathogens are able to produce fungal disease, systemic infection, and even death in the worst scenarios.

In the last century, fungal infection cases have dramatically increased, especially in developed countries. One work has shown that the number of cases of sepsis produced by fungal organisms in the USA has increased by 207\% between 1979 and 2000 (Martin et al., 2003). This phenomenon is associated with the appearance of medical techniques, such as the use of broad-spectrum antibiotics, the use of intravenous catheters, how intensive care units are organized, increased number of organ transplants, or the development of cytotoxic chemotherapies. On top of that, pandemics like HIV/AIDS have exponentially increased the number of patients with impaired immunity. In fact, fungal disease was extremely rare before all these changes occurred.

A paradigm of an emerging fungal organism is the yeast Saccharomyces cerevisiae. This species can be found naturally in many niches in the environment, but is most commonly known for its 
role as "baker's yeast" in either traditional or industrial fermentative production of bread, beer or wine. It has also been used as an agent to treat antibiotic-related diarrhea and as a nutritional supplement, when it is commercialized as $S$. boulardii. Classically, $S$. cerevisiae has been considered a safe non pathogenic organism. However in the last two decades, the number of cases of diagnosed infections has increased, probably as a result of the increased numbers of immunocompromised patients, but also due to advances made in diagnostic methodologies in hospitals, including genetic identification by molecular techniques. S. cerevisiae has been related to a wide variety of infections, which range from vaginitis in healthy patients and cutaneous infections, to systemic bloodstream infections and infections of essential organs in immunocompromised and critically ill patients (EnacheAngoulvant and Hennequin, 2005; Muñoz et al., 2005; de Llanos et al., 2011). Infected patients tend to be elderly people, premature children or patients suffering from immunosuppression due to HIV/AIDS, treatment with immunosuppressive agents, or other conditions associated with a deficient immune response. Furthermore, severe infections with $S$. cerevisiae have been occasionally reported in patients with no obvious predisposing factors (Jensen and Smith, 1976; Smith et al., 2002). All these data have changed the status of $S$. cerevisiae, which is now considered an emerging opportunistic pathogen (Herbrecht and Nivoix, 2005; de Llanos et al., 2006).

\section{S. cerevisiae POPULATION DIVERSITY: OPPORTUNISTIC STRAINS}

The species $S$. cerevisiae is very heterogeneous and contains strains with specific abilities like sherry wine strains, S. boulardii or baker strains. Before the development of high throughput sequencing techniques, the population structure of $S$. cerevisiae was not very clear. Now we know that it is structured into several genetically pure subpopulations and many mosaic strains that contain gene alleles of different subpopulations (Liti et al., 2009). In the last decade, yeast scientists have attempted to determine if the strains isolated from infected patients form a specific $S$. cerevisiae subpopulation with any special characteristic. de Llanos et al. (2004) used molecular markers as mt DNA restriction patterns and showed that clinical strains were present in several genetically differentiated groups of strains. In contrast, Carreto et al. (2008) used comparative genome hybridization on array $(\mathrm{aCGH})$, and suggested that clinical strains could be a genetically homogenous subpopulation. Later, Wei et al. (2007) sequenced the genome of $S$. cerevisiae strain YJM789 derived from a yeast isolated from the lung of an AIDS patient with pneumonia. Liti et al. (2009) sequenced 36 new strains that contained six clinical isolates. Strope et al. (2015) sequenced 93 strains from multiple geographic and environmental origins, including several clinical strains. Finally, after the sequencing of the whole genome of several clinical strains, it turns out that they are not a genetically homogenous group of strains, but are all mosaic strains with relatively heterogeneous genetic content (Liti et al., 2009; Strope et al., 2015).
Several studies have analyzed the potential virulence of this yeast species in vitro (McCusker et al., 1994; de Llanos et al., 2006) and in vivo (McCullough et al., 1998; de Llanos et al., 2011), and have suggested that some strains have the potential to cause disease regardless of their clinical or non clinical isolation origin. Many strains of S. cerevisiae, which have been isolated in clinical settings, present very low levels of virulence in mice infection models, while other strains, such as strain D14 isolated from a dietetic supplement, have shown a relative high level of virulence in different infection models (Llopis et al., 2012, 2014; Pérez-Torrado et al., 2015). Thus we propose that the term "opportunistic strain" is used more accurately and usefully to categorize these strains rather than "clinical strains" since not all clinical strains cause infections. Even more importantly, some non clinical strains can cause infections. We define opportunistic $S$. cerevisiae strains as those strains which show physiologic characteristics of yeast pathogens, such as growth, at $37{ }^{\circ} \mathrm{C}$ (McCusker et al., 1994; de Llanos et al., 2006), but unlike most other strains, can also cause infections and kill mice (de Llanos et al., 2011). These strains also survive better in human blood infection models than other strains, which may enable them to disseminate across the body and reach organs under adequate propagation conditions and in certain circumstances (Lin et al., 2012). Increased blood survival can be a key feature to distinguish these opportunistic yeasts strains from others.

\section{S. cerevisiae INFECTION MECHANISMS}

The infection mechanism of C. albicans, a fungal pathogen that is phylogenetically close to $S$. cerevisiae, is based on a first step of adhesion to human surface tissues and uses a family of proteins called adhesins. C. albicans is also able to penetrate epithelial or endothelial barriers via active mechanisms (Dalle et al., 2010). A second important aspect of infection mechanisms is resistance to the stressful situations generated after an encounter with cells of the immune system. In contrast, S. cerevisiae lacks homologous genes for the well-known C. glabrata or C. albicans adhesins and shows low adhesion levels to human tissues compared to both Candida species (Pérez-Torrado et al., 2012). It has been suggested that $S$. cerevisiae can only perform opportunistic or passive crossings when epithelial barrier integrity is previously compromised (Pérez-Torrado et al., 2012).

However, previous studies by our group (Llopis et al., 2012) have demonstrated that opportunistic $S$. cerevisiae strains show a specific transcription pattern after human blood infection, which reflects a specific oxidative stress response, increased amino acid biosynthesis and a DNA damage repair response (Figure 1).

Yeast pathogens are adapted to resist human defenses and one of the main responses of the immune system is microbe engulfment and oxidative burst. Neutrophils, macrophages and other cells with phagocytic capacity generate potent reactive oxygen and nitrogen species (ROS and RNS), which can be lethal to most fungal pathogens by causing damage to DNA, proteins and lipids (Bogdan et al., 2000). Most fungal pathogens display resistance to the reactive oxygen and nitrogen species 


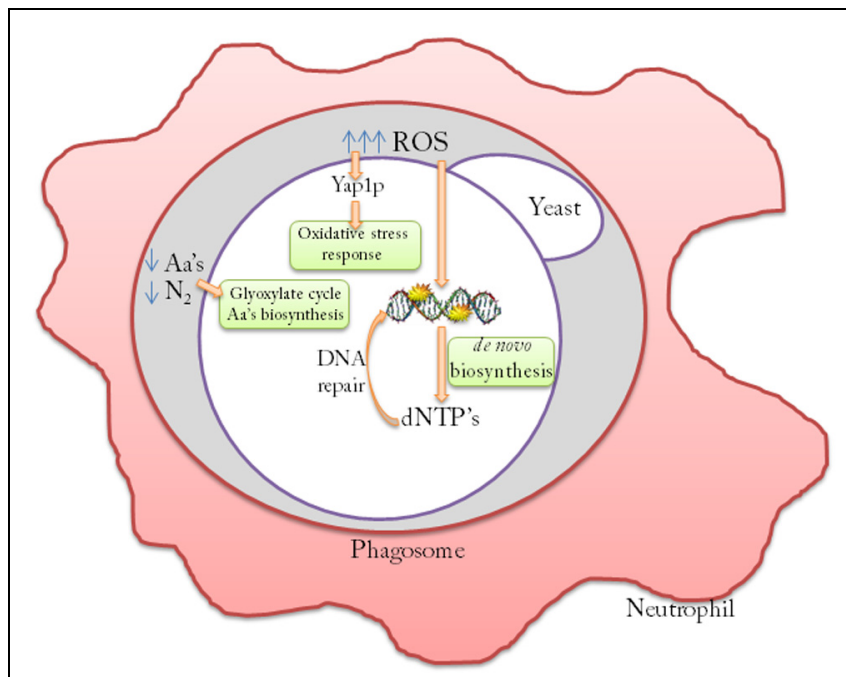

FIGURE 1 | Molecular mechanisms of persistence of Saccharomyces cerevisiae cells after neutrophil engulfment. Opportunistic $S$. cerevisiae strains redirect the low levels of the nitrogen sources inside the phagosome to increase amino acids (aa's) biosynthesis. These strains also increase the oxidative stress response by activating the Yap1p transcription factor regulon to counteract the lethal effects of reactive oxygen species (ROS) generated by the macrophage. De novo dNTP biosynthesis is activated to allow DNA repair machinery to counteract ROS-induced mutations.

used by human cells to counteract infection (Brown et al., 2009). Fungal resistance to ROS offers protection from oxidative host defenses and is undoubtedly an advantageous pathobiological property (Lushchak et al., 2010; Rodrigues-Pousada et al., 2010). It has been described that pathogens such as C. albicans or C. neoformans have the potential to resist, among other suboptimal conditions, oxidative stress produced by the ROS generated in the phagosome (Brown et al., 2007, 2014). Indeed the thioredoxin system of C. albicans and S. cerevisiae has been shown to be expressed during growth in human blood or mucosal tissue (Fradin et al., 2003, 2005, Zakikhany et al., 2007; Llopis et al., 2012), which indicates that the ability to respond to oxidative stress might be crucial in early stages of systemic infections. TRX1 (thioredoxin 1) is also necessary to survive the oxidative environment of macrophages in C. neoformans, and is important for the virulence of this fungal pathogen (Missall and Lodge, 2005). The TSA2 (thioredoxin peroxidase 2) and GPX2 (glutathione peroxidase 2) genes have been shown to be induced in $S$. cerevisiae strains when exposed to neutrophils (RubinBejerano et al., 2003), and a clear antioxidant response has been observed. Fradin et al. (2005) demonstrated that neutrophils play a key role in bloodstream infections with C. albicans. This observation is in line with the high susceptibility shown by neutropenic patients (deficient in these immune cells) to disseminated candidiasis (Bodey et al., 1992; Wright and Wenzel, 1997). In S. cerevisiae, the main role of proper oxidative stress response virulence has been suggested since a virulent strain mutant in transcription factor Yap1p, the main transcription factor involved in oxidative stress response that is unable to grow under oxidative stress conditions, presented low survival levels in human blood compared with the wild type or the YAP1 reconstituted strain (Llopis et al., 2012). Diezmann and Dietrich (2011) compared hundreds of clinical isolates and showed that they were more resistant to oxidative stress after they verified the central role of oxidative stress resistance in S. cerevisiae virulence.

After exposure to human neutrophils or cultured macrophages, $C$. albicans cells up-regulate amino acid biosynthetic genes (Rubin-Bejerano et al., 2003; Fradin et al., 2005). Rubin-Bejerano et al. (2003) observed induction for these pathways after $C$. albicans and $S$. cerevisiae cells were ingested by neutrophils. This suggests that the microenvironment in the phagosome inside the neutrophil is deficient in amino acids, and generates a rapid response from yeast strains. The methionine and arginine biosynthetic genes are induced when $S$. cerevisiae is phagocytized by the murine macrophage-like cell line (Lorenz and Fink, 2001). Kingsbury et al. (2006) revealed the relevance of amino acid biosynthesis for yeast survival in a murine host and suggested that yeast can use a variety of nitrogen sources under these conditions. The glyoxylate cycle is also induced upon phagocytes ingestion of the bacterium Mycobacterium tuberculosis (McKinney et al., 2000), and other fungi such as C. neoformans (Rude et al., 2002), Leptosphaeria maculans (Idnurm and Howlett, 2002), C. albicans (Lorenz and Fink, 2001), and S. cerevisiae (Lorenz and Fink, 2001). This change in metabolism is a response to the glucose-poor environment of the macrophage, and contributes to the virulence of some pathogens. The ICL1 gene, which encodes for isocitrate lyase, one of the principal enzymes of the glyoxylate cycle, has been recently shown to be substantially induced upon exposure to macrophages in vitro in both S. cerevisiae and C. albicans (Lorenz and Fink, 2001; Lorenz et al., 2004). All these data suggest that the ICL1 gene may also play a general role in S. cerevisiae in human infections.

A common mechanism described for human microbial pathogens is to increase dNTP pools in order to repair the DNA damage caused by the oxidative burst of phagocytes. The importance of a de novo nucleotide biosynthetic pathway for phagocyte survival has been demonstrated for bacterial pathogens such as Salmonella enterica (Panosa et al., 2010), Bacteroides fragilis (Smalley et al., 2002), Pseudomonas aeruginosa (Sjöberg and Torrents, 2011), Staphylococcus aureus (Kirdis et al., 2007), Streptococcus pyogenes (Le Breton et al., 2013), Bacillus anthracis, and Escherichia coli (Samant et al., 2008). The relevance of de novo nucleotide biosynthetic pathways has also been implicated for fungal pathogens like Cryptococcus neoformans (Morrow et al., 2012) or C. albicans (Donovan et al., 2001; Jiang et al., 2010), and nucleotide biosynthetic pathways have been discussed as a target for antifungal compounds (Rodriguez-Suarez et al., 2007). In a recent study, we observed that $S$. cerevisiae opportunistic strains, like other human pathogens, have the enhanced ability to produce dNTPs, the substrates used by DNA repair machineries (Pérez-Torrado et al., 2015). The importance of this pathway for the virulence of $S$. cerevisiae has been confirmed by experimental infections conducted in immunodeficient murine models using a $\Delta$ gual mutant, which is a key enzyme for de novo dNTP biosynthesis. Additions of 
exogenous guanine and the use of mutants in the DNA damage checkpoint, which activates dNTP biosynthesis in yeast cells, affects the survival of yeast cells in ex vivo blood infections (PérezTorrado et al., 2015). The nitrogen source preferentially used by yeasts in phagosomes to increase this pathway is still unknown.

\section{CONCLUSION AND PERSPECTIVES}

In developed countries, consumers have been driven to take a more critical attitude about what they eat and drink as a requirement of modern life. Food microbiologists are facing the huge challenge of regarding food freshness that is implicit in consumer demand for more natural products. Additive-free safer food with less severe processing that has a satisfactory shelf life and is easy to prepare is required given the greater awareness of nutrition and health. These changes in consumer preferences, which modify food processes, may have important consequences and could affect both food quality and safety. Yeasts, especially S. cerevisiae, have an impeccably good food safety record compared to other microorganisms like virus, bacteria and some filamentous fungi. However, humans unknowingly and inadvertently ingest large, viable

\section{REFERENCES}

Bodey, G., Bueltmann, B., Duguid, W., Gibbs, D., Hanak, H., Hotchi, M., et al. (1992). Fungal infections in cancer patients: an international autopsy survey. Eur. J. Clin. Microbiol. Infect. Dis. 11, 99-109. doi: 10.1007/BF0196 7060

Bogdan, C., Rollinghoff, M., and Diefenbach, A. (2000). Reactive oxygen and reactive nitrogen intermediates in innate and specific immunity. Curr. Opin. Immunol. 12, 64-76. doi: 10.1016/S0952-7915(99)00052-7

Brown, A. J., Budge, S., Kaloriti, D., Tillmann, A., Jacobsen, M. D., Yin, Z., et al. (2014). Stress adaptation in a pathogenic fungus. J. Exp. Biol. 217, 144-155. doi: 10.1242/jeb.088930

Brown, A. J., Haynes, K., and Quinn, J. (2009). Nitrosative and oxidative stress responses in fungal pathogenicity. Curr. Opin. Microbiol. 12, 384-391. doi: 10.1016/j.mib.2009.06.007

Brown, S. M., Campbell, L. T., and Lodge, J. K. (2007). Cryptococcus neoformans, a fungus under stress. Curr. Opin. Microbiol. 10, 320-325. doi: 10.1016/j.mib.2007.05.014

Carreto, L., Eiriz, M. F., Gomes, A. C., Pereira, P. M., Schuller, D., and Santos, M. A. (2008). Comparative genomics of wild type yeast strains unveils important genome diversity. BMC Genomics 9:524. doi: 10.1186/1471-216 4-9-524

Dalle, F., Wachtler, B., L'Ollivier, C., Holland, G., Bannert, N., Wilson, D., et al. (2010). Cellular interactions of Candida albicans with human oral epithelial cells and enterocytes. Cel. Microbiol. 12, 248-271. doi: 10.1111/j.14625822.2009.01394.x

de Llanos, R., Fernandez-Espinar, M. T., and Querol, A. (2006). A comparison of clinical and food Saccharomyces cerevisiae isolates on the basis of potential virulence factors. Antonie Van Leeuwenhoek 90, 221-231. doi: 10.1007/s10482006-9077-7

de Llanos, R., Llopis, S., Molero, G., Querol, A., Gi, C., and Fernandez-Espinar, M. T. (2011). In vivo virulence of commercial Saccharomyces cerevisiae strains with pathogenicity-associated phenotypical traits. Int. J. Food. Microbiol. 144, 393-399. doi: 10.1016/j.ijfoodmicro.2010.10.025

de Llanos, R., Querol, A., Planes, A. M., and Fernández-Espinar, M. T. (2004). Molecular characterization of clinical Saccharomyces cerevisiae isolates and their association with non-clinical strains. Syst. Appl. Microbiol. 27, 427-435. doi: $10.1078 / 0723202041438473$ populations of S. cerevisiae without them having adverse impacts on their health (e.g., yeasts in home-brewed beer, beers enriched by yeast or dietary supplements that contain yeast are very common today). Nevertheless, having an open mind about, and conducting vigilance of, yeasts and food-borne diseases are required. Compared with other microbial groups, yeasts are not seen as aggressive pathogens, but they are capable of causing human disease in opportunistic circumstances (PuigAsensio et al., 2014). As we summarize in this paper, yeast researchers have made progress in understanding the nature and virulence mechanism in $S$. cerevisiae strains. In the future, more attention has to be paid to industrial practices that are more prone to generate opportunistic S. cerevisiae strains.

\section{ACKNOWLEDGMENTS}

RP-T was supported by CICYT grants (ref. AGL2012-39937CO2-01 and -02) from the Spanish Ministry of Education and Science and FEDER. This work was supported by grant PROMETEO (project PROMETEO/2009/019) from the Generalitat Valenciana.

Diezmann, S., and Dietrich, F. S. (2011). Oxidative stress survival in a clinical Saccharomyces cerevisiae isolate is influenced by a major quantitative trait nucleotide. Genetics 188, 709-722. doi: 10.1534/genetics.111.128256

Donovan, M., Schumuke, J. J., Fonzi, W. A., Bonar, S. L., Gheesling-Mullis, K., Jacob, G. S., et al. (2001). Virulence of a phosphoribosyl aminoimidazole carboxylase-deficient Candida albicans strain in an immunosuppressed murine model of systemic candidiasis. Infect. Immun. 69, 2542-2548. doi: 10.1128/IAI.69.4.2542-2548.2001

Enache-Angoulvant, A., and Hennequin, C. (2005). Invasive Saccharomyces infection: a comprehensive review. Clin. Infect. Dis. 41, 1559-1568. doi: $10.1086 / 497832$

Fradin, C., De Groot, P., MacCallum, D., Schaller, M., Klis, F., Odds, F. C., et al. (2005). Granulocytes govern the transcriptional response, morphology and proliferation of Candida albicans in human blood. Mol. Microbiol. 56, 397-415. doi: 10.1111/j.1365-2958.2005.04557.x

Fradin, C., Kretschmar, M., Nichterlein, T., Gaillardin, C., d'Enfert, C., and Hube, B. (2003). Stage-specific gene expression of Candida albicans in human blood. Mol. Microbiol. 47, 1523-1543. doi: 10.1046/j.1365-2958.2003. 03396.x

Herbrecht, R., and Nivoix, Y. (2005). Saccharomyces cerevisiae fungemia: an adverse effect of Saccharomyces boulardii probiotic administration. Clin. Infect. Dis. 40, 1635-1637. doi: 10.1086/429926

Idnurm, A., and Howlett, B. J. (2002). Isocitrate lyase is essential for pathogenicity of the fungus Leptosphaeria maculans to canola (Brassica napus). Eukaryot. Cell 1, 719-724. doi: 10.1128/EC.1.5.719-724.2002

Jensen, D. P., and Smith, D. L. (1976). Fever of unknown origin secondary to brewer's yeast ingestion. Arch. Intern. Med. 136, 332-333. doi: 10.1001/archinte.1976.03630030064011

Jiang, L., Zhao, J., Guo, R., Li, J., Yu, L., and Xu, D. (2010). Functional characterization and virulence study of ADE8 and GUA1 genes involved in the de novo purine biosynthesis in Candida albicans. FEMS Yeast Res. 10, 199-208. doi: 10.1111/j.1567-1364.2009.00600.x

Kingsbury, J. M., Goldstein, A. L., and McCusker, J. H. (2006). Role of nitrogen and carbon transport, regulation, and metabolism genes for Saccharomyces cerevisiae survival in vivo. Eukaryot. Cell 5, 816-824. doi: 10.1128/EC.5.5.816824.2006

Kirdis, E., Jonsson, I. M., Kubica, M., Potempa, J., Josefsson, E., and Masalha, M. (2007). Ribonucleotide reductase class III, an essential enzyme for the anaerobic 
growth of Staphylococcus aureus, is a virulence determinant in septic arthritis. Microb. Pathog. 3, 179-188. doi: 10.1016/j.micpath.2007.05.008

Le Breton, Y., Mistry, P., Valdes, K. M., Quigley, J., Kumar, N., Tettelin, H., et al. (2013). Genome-wide identification of genes required for fitness of group a Streptococcus in human blood. Infect. Immun. 81, 862-875. doi: 10.1128/IAI.00837-12

Lin, L., Ibrahim, A. S., Baquir, B., Palosaari, A., and Spellberg, B. (2012). Luminescent-activated transfected killer cells to monitor leukocyte trafficking during systemic bacterial and fungal infection. J. Infect. Dis. 205, 337-347. doi: 10.1093/infdis/jir72522124127

Liti, G., Carter, D. M., Moses, A. M., Warringer, J., Parts, L., James, S. A., et al. (2009). Population genomics of domestic and wild yeasts. Nature 458, 337-341. doi: $10.1038 /$ nature 07743

Llopis, S., Hernández-Haro, C., Monteoliva, L., Querol, A., Molina, M., and Fernández-Espinar, M. T. (2014). Pathogenic potential of Saccharomyces strains isolated from dietary supplements. PLoS ONE 9:e98094. doi: 10.1371/journal.pone.0098094

Llopis, S., Querol, A., Heyken, A., Hube, B., Jespersen, L., Fernandez-Espinar, T., et al. (2012). Transcriptomics in human blood incubation reveals the importance of oxidative stress response in Saccharomyces cerevisiae clinical strains. BMC Genomics 13:419. doi: 10.1186/1471-2164-13-419

Lorenz, M. C., Bender, J. A., and Fink, G. R. (2004). Transcriptional response of Candida albicans upon internalization by macrophages. Eukaryot. Cell 3, 1076-1087. doi: 10.1128/EC.3.5.1076-1087.2004

Lorenz, M. C., and Fink, G. R. (2001). The glyoxylate cycle is required for fungal virulence. Nature 412, 83-86. doi: 10.1038/35083594

Lushchak, O. V., Inoue, Y., and Lushchak, V. I. (2010). Regulatory protein Yap1 is involved in response of yeast Saccharomyces cerevisiae to nitrosative stress. Biochemistry (Mosc.) 75, 629-664. doi: 10.1134/S00062979100 50135

Martin, G. S., Mannino, D. M., Eaton, S., and Moss, M. (2003). The epidemiology of sepsis in the United States from 1979 through 2000. N. Engl. J. Med. 348, 1546-1554. doi: 10.1056/NEJMoa022139

McCullough, M. J., Clemons, K. V., McCusker, J. H., and Stevens, D. A. (1998). Species identification and virulence attributes of Saccharomyces boulardii (nom. inval.). J. Clin. Microbiol. 36, 2613-2617.

McCusker, J. H., Clemons, K. V., Stevens, D. A., and Davis, R. W. (1994). Saccharomyces cerevisiae virulence phenotype as determined with CD-1 mice is associated with the ability to grow at 42 degrees $C$ and form pseudohyphae. Infect. Immun. 62, 5447-5455.

McKinney, J. D., Honerzu, B. K., Munoz-Elias, E. J., Miczak, A., Chen, B., Chan, W. T., et al. (2000). Persistence of Mycobacterium tuberculosis in macrophages and mice requires the glyoxylate shunt enzyme isocitrate lyase. Nature 406, 735-738. doi: 10.1038/35021074

Missall, T. A., and Lodge, J. K. (2005). Function of the thioredoxin proteins in Cryptococcus neoformans during stress or virulence and regulation by putative transcriptional modulators. Mol. Microbiol. 57, 847-858. doi: 10.1111/j.13652958.2005.04735.x

Morrow, C. A., Valkov, E., Stamp, A., Chow, E. W., Lee, I. R., Wronski, A., et al. (2012). De novo GTP biosynthesis is critical for virulence of the fungal pathogen Cryptococcus neoformans. PLoS Pathog. 8:e1002957. doi: 10.1371/journal.ppat.1002957

Muñoz, P., Bouza, E., Cuenca-Estrella, M., Eiros, J. M., Perez, M. J., SanchezSomolinos, M., et al. (2005). Saccharomyces cerevisiae fungemia: an emerging infectious disease. Clin. Infect. Dis. 40, 1625-1634. doi: 10.1086/429916

Panosa, A., Roca, I., and Gibert, I. (2010). Ribonucleotide reductases of Salmonella typhimurium transcriptional regulation and differential role in pathogenesis. PLoS ONE 5:e11328. doi: 10.1371/journal.pone.0011328

Pérez-Torrado, R., Llopis, S., Jespersen, L., Fernández-Espinar, T., and Querol, A. (2012). Clinical Saccharomyces cerevisiae isolates cannot cross the epithelial barrier in vitro. Int. J. Food Microbiol. 157, 59-64. doi: 10.1016/j.ijfoodmicro.2012.04.012
Pérez-Torrado, R., Llopis, S., Perrone, B., Gómez-Pastor, R., Hube, B., and Querol, A. (2015). Comparative genomic analysis reveals a critical role of de novo nucleotide biosynthesis for Saccharomyces cerevisiae virulence. PLoS ONE 10:e0122382. doi: 10.1371/journal.pone.0122382

Pfaller, M. A., and Diekema, D. J. (2007). Epidemiology of invasive candidiasis: a persistent public health problem. Clin. Microbiol. Rev. 20, 133-163. doi: 10.1128/CMR.00029-06

Puig-Asensio, M., Padilla, B., Garnacho-Montero, J., Zaragoza, O., Aguado, J. M., Zaragoza, R., et al. (2014). Epidemiology and predictive factors for early and late mortality in Candida bloodstream infections: a population-based surveillance in Spain. Clin. Microbiol. Infect. 20, O245-O254. doi: 10.1111/1469-0691.12380

Rodrigues-Pousada, C., Menezes, R. A., and Pimentel, C. (2010). The Yap family and its role in stress response. Yeast 27, 245-258. doi: 10.1002/yea.1752

Rodriguez-Suarez, R., Xu, D., Veillette, K., Davison, J., Sillaots, S., Kauffman, S., et al. (2007). Mechanism-of-action determination of GMP synthase inhibitors and target validation in Candida albicans and Aspergillus fumigatus. Chem. Biol. 14, 1163-1175. doi: 10.1016/j.chembiol.2007.09.009

Rubin-Bejerano, I., Fraser, I., Grisafi, P., and Fink, G. R. (2003). Phagocytosis by neutrophils induces an amino acid deprivation response in Saccharomyces cerevisiae and Candida albicans. Proc. Natl. Acad. Sci. U.S.A. 100, 11007-11012. doi: $10.1073 /$ pnas. 1834481100

Rude, T. H., Toffaletti, D. L., Cox, G. M., and Perfect, J. R. (2002). Relationship of the glyoxylate pathway to the pathogenesis of Cryptococcus neoformans. Infect. Immun. 70, 5684-5694. doi: 10.1128/IAI.70.10.5684-5694.2002

Samant, S., Lee, H., Ghassemi, M., Chen, J., Cook, J. L., Mankin, A. S., et al. (2008). Nucleotide biosynthesis is critical for growth of bacteria in human blood. PLoS Pathog. 4:e37. doi: 10.1371/journal.ppat.0040037

Sjöberg, B. M., and Torrents, E. (2011). Shift in ribonucleotide reductase gene expression in Pseudomonas aeruginosa during infection. Infect. Immun. 79, 2663-2669. doi: 10.1128/IAI.01212-10

Smalley, D., Rocha, E. R., and Smith, C. J. (2002). Aerobic-type ribonucleotide reductase in the anaerobe Bacteroides fragilis. J. Bacteriol. 184, 895-903. doi: 10.1128/jb.184.4.895-903.2002

Smith, D., Metzgar, D., Wills, C., and Fierer, J. (2002). Fatal Saccharomyces cerevisiae aortic graft infection. J. Clin. Microbiol. 40, 2691-2692. doi: 10.1128/JCM.40.7.2691-2692.2002

Strope, P. K., Skelly, D. A., Kozmin, S. G., Mahadevan, G., Stone, E. A., Magwene, P. M., et al. (2015). The 100-genomes strains, an S. cerevisiae resource that illuminates its natural phenotypic and genotypic variation and emergence as an opportunistic pathogen. Genome Res. 25, 762-774. doi: 10.1101/gr.185538.114

Wei, W., McCusker, J. H., Hyman, R. W., Jones, T., Ning, Y., Cao, Z., et al. (2007). Genome sequencing and comparative analysis of Saccharomyces cerevisiae strain YJM789. Proc. Natl. Acad. Sci. U.S.A. 104, 12825-12830. doi: 10.1073/pnas.0701291104

Wright, W. L., and Wenzel, R. P. (1997). Nosocomial candida epidemiology, transmission, and prevention. Infect. Dis. Clin. North. Am. 11, 411-425. doi: 10.1016/S0891-5520(05)70363-9

Zakikhany, K., Naglik, J. R., Schmidt-Westhausen, A., Holland, G., Schaller, M., and Hube, B. (2007). In vivo transcript profiling of Candida albicans identifies a gene essential for interepithelial dissemination. Cell. Microbiol. 9, 2938-2954. doi: 10.1111/j.1462-5822.2007.01009.x

Conflict of Interest Statement: The authors declare that the research was conducted in the absence of any commercial or financial relationships that could be construed as a potential conflict of interest.

Copyright (c) 2016 Pérez-Torrado and Querol. This is an open-access article distributed under the terms of the Creative Commons Attribution License (CC BY). The use, distribution or reproduction in other forums is permitted, provided the original author(s) or licensor are credited and that the original publication in this journal is cited, in accordance with accepted academic practice. No use, distribution or reproduction is permitted which does not comply with these terms. 